\title{
Abnormal Bolus Reflux Is Associated With Poor Pulmonary Outcome in Patients With Idiopathic Pulmonary Fibrosis
}

\author{
Lawrence F Borges, ${ }^{1,2}$ Vikrant Jagadeesan, ${ }^{2,3}$ Hilary Goldberg, ${ }^{2,4}$ Sravanya Gavini, ${ }^{5}$ Wai-Kit Lo, ${ }^{1,2,6}$ Robert Burakoff, ${ }^{1,2}$ \\ Natan Feldman, ${ }^{1}$ and Walter W Chan ${ }^{1,2 *}$ \\ ${ }^{I}$ Division of Gastroenterology, Hepatology and Endoscopy, Brigham and Women's Hospital, Boston, MA, USA, ${ }^{2}$ Harvard Medical School, Boston, \\ MA, USA; ${ }^{3}$ Department of Medicine, Brigham and Women's Hospital, Boston, MA, USA; ${ }^{4}$ Division of Pulmonary Medicine, Brigham and Women's \\ Hospital, Boston, MA, USA; ${ }^{5}$ Division of Gastroenterology, University of Texas Southwestern Medical Center, Dallas, TX, USA; and ${ }^{6}$ Department of \\ Gastroenterology, Boston VA Healthcare System, Boston, MA, USA
}

\section{Background/Aims}

Gastroesophageal reflux (GER) is postulated to play a role in idiopathic pulmonary fibrosis (IPF). However, the value of objective GER measures in predicting IPF disease progression is unclear. We aim to evaluate the association between objective GER measures on multichannel intraluminal impedance and $\mathrm{pH}(\mathrm{MII}-\mathrm{pH})$ testing and development of poor pulmonary outcomes within 1 year in prelung transplant IPF patients.

\section{Methods}

This was a retrospective cohort study of adults with IPF who underwent pre-lung transplant evaluation with MII-pH off proton pump inhibitors (PPI) at a tertiary care center from June 2008 to November 2015. Patients were followed for 1 year from time of MII-pH for poor pulmonary outcomes, defined by hospitalization for respiratory exacerbation or death. Univariate, multivariate and time-to-event analyses were performed to assess associations between baseline GER parameters on MII-pH and poor outcomes.

\section{Results}

Eighty-four subjects (mean age 61.1 years, 64.3\% male) were included. Subjects with increased bolus exposure time (BET) had a higher incidence of 1 -year poor pulmonary outcome vs normal BET (45.7\% vs $15.2 \%, P=0.006)$. Increased BET remained an independent predictor of poor outcome after controlling for age, gender, body mass index, smoking, lung disease severity, and PPI use $(\mathrm{OR}, 4.18 ; P=0.030)$. Increased BET was also predictive of decreased time to poor pulmonary outcome (hazard ratio $[H R], 4.88 ; P=$ 0.007). Subgroup analyses showed that increased BET remained independently associated with time to pulmonary hospitalization (HR, 4.00; $P=0.020)$, with a trend for 1 -year mortality $(H R, 2.19 ; P=0.380)$.

\section{Conclusion}

Increased BET on MII-pH is an independent predictor of poor pulmonary outcome over 1 year in IPF patients.

(J Neurogastroenterol Motil 2018;24:395-402)

\section{Key Words}

Aspiration; Extra-esophageal reflux; Gastroesophageal reflux; Lung disease; Outcomes

\section{Received: January 14, 2018 Revised: April 8, 2018 Accepted: April 26, 2018}

(a) This is an Open Access article distributed under the terms of the Creative Commons Attribution Non-Commercial License (http://creativecommons. org/licenses/by-nc/4.0) which permits unrestricted non-commercial use, distribution, and reproduction in any medium, provided the original work is properly cited.

${ }^{*}$ Correspondence: Walter W Chan, MD, MPH Division of Gastroenterology, Hepatology and Endoscopy, Brigham and Women's Hospital, 75 Francis Street, Boston, MA 02115 , USA

Tel: +1-617-732-6389, Fax: +1-617-525-0338, E-mail: wwchan@bwh.harvard.edu 


\section{Introduction}

Idiopathic pulmonary fibrosis (IPF) is a progressive, unpredictable, and almost universally fatal lung disease without lung transplantation. Gastroesophageal reflux (GER) has been associated with $\mathrm{IPF}^{1}$ and postulated to play a role in IPF pathogenesis; however, the exact nature of the relationship between GER and IPF remains unclear. One proposed mechanism suggests that refluxed contents from the stomach and esophagus may be introduced into the lungs via micro-aspiration, thereby triggering an inflammatory cascade that ultimately results in fibrotic lung injury. On the other hand, altered respiratory mechanics resulting from IPF itself may worsen GER through alteration of the trans-diaphragmatic pressure gradient. Therefore, the directionality of the association between GER and IPF remains debated.

Previous studies have established the high prevalence of both acid and non-acid reflux in patients with $\mathrm{IPF}^{1,2}$ It has also been demonstrated that IPF patients have more abnormal reflux than patients with non-fibrotic lung disease of similar clinical severity. ${ }^{3}$ These findings strengthen the argument that GER may be a modifiable risk factor in the pathogenesis of IPF. However, a direct causal relationship between GER and IPF could not be conclusively established through these cross-sectional studies. Indeed, a clear association between abnormal GER and IPF disease progression has never been demonstrated. An understanding of the linear relationship between GER and IPF disease outcome may provide evidence for GER intervention as a treatment strategy for IPF, and establish the utility of objective GER testing in managing IPF. In this study, we aim to evaluate the association between objective measures of GER on 24-hour multichannel intraluminal impedance and $\mathrm{pH}$ (MII-pH) study, and the development of poor pulmonary outcomes over 1 year in IPF patients.

\section{Materials and Methods}

\section{Subject Selection}

This was a retrospective cohort study of adult subjects (age $>$ 18 years) with IPF who underwent MII-pH as part of standard pre-lung transplant evaluation between June 2008 and September 2015 at a tertiary care center. The indication for MII-pH for all patients was consideration of lung transplant listing, regardless of the presence or history of any reflux symptoms. The pulmonary diagnosis of IPF was made in these subjects if they met at least one of the following 3 criteria, consistent with American Thoracic Society guideline recommendations ${ }^{4}$ : (1) lung biopsy consistent with usual interstitial pneumonitis; (2) lung biopsy consistent with possible usual interstitial pneumonitis or non-specific interstitial pneumonitis; or, (3) in the absence of biopsy data, the subject was felt to meet criteria for the diagnosis of IPF by their pulmonologist based on characteristic clinical and radiologic findings.

To standardize the assessment of reflux, patients were excluded from the study if they had previously undergone anti-reflux surgery, or if they had taken acid suppression therapy within 7 days of MII$\mathrm{pH}$. Baseline characteristics at the time of MII-pH, such as age, sex, body mass index (BMI), smoking history, and history of proton pump inhibitor (PPI) use were recorded. To assess baseline lung disease severity, pulmonary function test measurements and oxygen use data were collected at a time closest to and within 3 months of MII-pH. Oxygen use data included the frequency of use (never vs intermittent vs continuous use) and flow rate (in liters per minute) at the time of MII-pH.

\section{Multichannel Intraluminal Impedance and pH Measurement}

Exposure to reflux was objectively assessed by MII-pH. All patients underwent MII-pH (Sandhill Scientific Inc, Highland Ranch, CO, USA) after an overnight fast and discontinuing acid suppression medication for at least 7 days. The MII-pH system consists of a portable electronic data logger and transnasal catheter equipped with $2 \mathrm{pH}$ electrodes ( 0 and $15 \mathrm{~cm}$ ), and 8 impedance electrodes $(-3,-1,1,3,5,9,11$, and $13 \mathrm{~cm})$. The catheter was placed into the esophagus and positioned with the distal $\mathrm{pH}$ electrode at $5 \mathrm{~cm}$ above the lower esophageal sphincter (LES). Distal or "total" reflux was detected at the most caudal electrode $5 \mathrm{~cm}$ from the LES, while "proximal" reflux was defined by events reaching the most cranial electrode, $15 \mathrm{~cm}$ above the LES. Over the course of the 24-hour study, subjects were instructed to remain upright during the day and recumbent at night, and maintain their normal activities, including meals. They were also asked to document meal periods via the data logger, and these periods were excluded from analysis. All MII-pH tracings were manually reviewed by 1 of 2 authors (W.W.C. and R.B.) who were trained and experienced in reading MII-pH and esophageal motility studies. MII-pH results were analyzed with the assistance of a dedicated software package (Bioview Analysis, version 5.6.3.0; Sandhill Scientific Inc). A minority of patients $(\mathrm{n}=33)$ in our study underwent MII-pH at an outside institution. The data from these outside studies were only included if the patient preparation and technique of administration were adequately similar to that of our institution and if their data/ tracing were available for review by the authors. 
Several measures of reflux severity as determined by MII-pH were recorded for each subject, including number of bolus reflux episodes (total and proximal), bolus reflux exposure time (ie, percentage of study time in reflux), acid exposure time (ie, percentage of study time with $\mathrm{pH}<4$ ), acid reflux episodes (total and proximal), and non-acid reflux episodes (total and proximal). Reflux episodes were defined as a 50\% drop in impedance from the baseline, progressing from distal to proximal impedance channels in a retrograde fashion. The duration, or bolus clearance time, of each reflux episode was defined as the time between the initial drop below $50 \%$ of baseline impedance, signaling onset of reflux, and the return to $50 \%$ of baseline in the distal impedance channel, signaling completion of the reflux event. Bolus exposure time (BET) was a percentage calculated by dividing the total bolus clearance time of all reflux episodes by the total analyzed study time (excluding meal times) (Fig. 1). Previously established 95th percentile values, derived from normal volunteers, were used to determine normal cutoffs for reflux episodes and exposure times, per widespread clinical use, ${ }^{5,6}$ and increased BET was defined as $\geq 1.4 \%$.

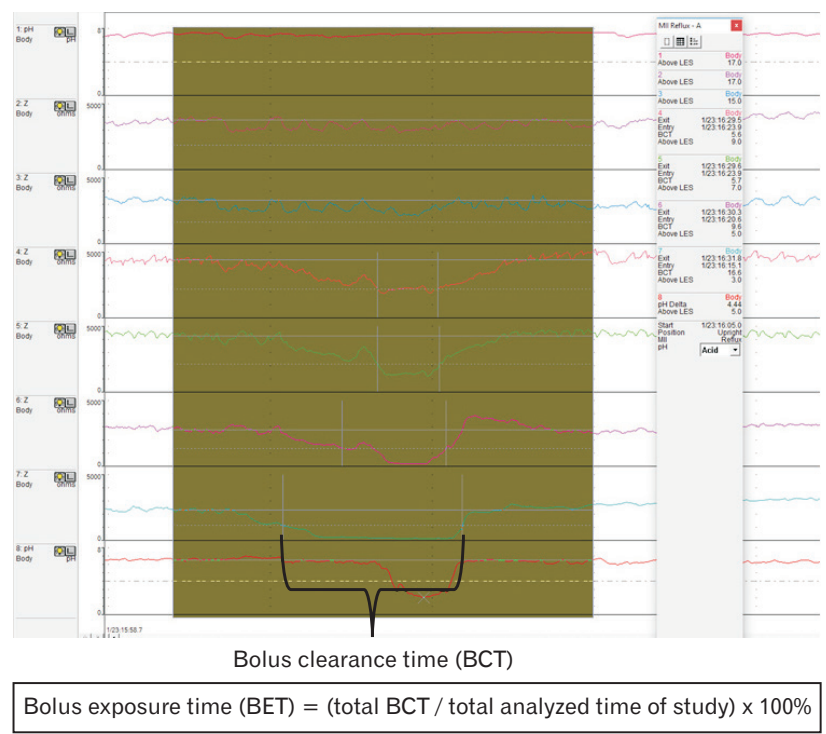

Figure 1. Representative image of an impedance reflux episode and the measurements taken to determine bolus exposure time (BET). Reflux episodes were defined as a 50\% drop in impedance from baseline, progressing from distal to proximal impedance channels in a retrograde fashion. Bolus clearance time (BCT) for each reflux episode was defined as the time between the initial drop below $50 \%$ of baseline impedance, signaling onset of reflux, and the return to $50 \%$ of baseline in the distal impedance channel, signaling completion of the reflux event. BET was a percentage calculated by dividing the total BCT of all reflux episodes by the total analyzed study time (excluding meal times).

\section{Outcome Measures}

The primary outcome of interest was the incidence of poor pulmonary outcomes, defined as either a hospitalization for exacerbation of respiratory disease or death, within one year of MII-pH testing. Sub-group analyses were also performed to evaluate hospitalization and mortality as separate outcomes. All hospitalization records during the follow-up period were manually reviewed, and only those directly related to exacerbation of underlying respiratory disease were included. Elective admissions for procedures or completion of transplantation work-up, or those in which the admitting diagnoses were unrelated to lung disease, were excluded. Reviewers of hospitalization records were blinded to the MII-pH results. In addition to incidence of poor outcome within 1 year of MII-pH, time-to-event analyses were performed to assess the effect of GER on time to occurrence of poor pulmonary outcome (hospitalization or death), hospitalization alone, and mortality alone, within 1 year of MII-pH testing.

\section{Statistical Methods}

The prevalence of developing poor pulmonary outcome within 1 year of MII-pH was compared between those with normal vs abnormal reflux. Univariate analyses were first conducted to look for significant associations between baseline GER parameters on MII-pH and the outcomes of interest. Dichotomous variables were analyzed using Fisher's exact test and continuous variables were analyzed using Student's $t$ test. Multivariate analyses were then performed using logistic regression to control for baseline characteristics and potential confounders. Time-to-event analyses were performed using Cox regression and Kaplan-Meier analyses to determine if reflux severity was associated with a decreased time to poor outcomes, with censoring at the time of anti-reflux surgery, lung transplant, or last clinic follow-up, whichever was earliest. All statistical analyses were performed using SAS v9.1 (SAS Institute Inc, Cary, NC, USA).

The study was approved by the Partners Healthcare Institutional Review Board (2011P001563). Due to the retrospective nature of the study and minimal risk, patient informed consent was waived for the study.

\section{Results}

\section{Demographics}

We identified a total of 84 subjects with IPF who met the inclusion criteria. The mean age at the time of MII-pH was 61.1 years 
(SD 8.66), and 54 (64.3\%) patients were men. Among these 84 patients, 73 had one full year of follow-up without undergoing antireflux surgery or lung transplantation. Of these, 18 (24.7\%) were hospitalized, and 8 (11.0\%) died during the 1-year study period. Overall, 5 subjects underwent anti-reflux surgery during the followup period, and 16 received a lung transplant.

Forty-six (54.8\%) subjects had abnormal reflux as determined by increased BET. Baseline characteristics at the time of MII-pH were assessed between subjects with and without abnormal reflux to address possible confounding risk factors for hospitalization and death (Table 1). Patients with abnormal BET were slightly younger (mean age $58.4 \pm 8.14$ years vs $63.1 \pm 8.94$ years, $P=0.050$ ) and had significantly higher prevalence of PPI use $(71.7 \%$ vs $36.8 \%, P$ $=0.001)$. No significant difference was observed in terms of gender, BMI, smoking history, or baseline lung disease severity.

For other reflux parameters obtained on MII-pH, 24 (28.6\%) subjects had abnormal acid exposure time (AET $>4.0 \%$ ), with a mean AET of $4.02 \%$ (SD 5.52) for the whole cohort. Nineteen (22.6\%) patients had abnormal AET in the upright position (upright AET > 6.3\%) and 29 (34.5\%) subjects had abnormal AET in the recumbent position (recumbent AET $>1.2 \%$ ). The overall mean upright AET and recumbent AET were 27.6\% (SD 53.20) and $3.8 \%$ (SD 6.13), respectively. Twenty-nine (34.5\%) patients had increased total reflux episodes ( $>73)$, with an overall mean total reflux episodes of 61.8 (SD 36.41).

\section{Multichannel Intraluminal Impedance and $\mathrm{pH}$ Measurement and 1-Year Incidence of Poor Pulmonary Outcome}

On univariate analyses, abnormally increased BET on MII$\mathrm{pH}$ was associated with higher incidence of poor pulmonary outcome within 1 -year vs normal BET ( $45.7 \%$ vs $15.2 \%, P=0.006$ ). Abnormal BET was also significantly associated with hospitalization only (44.1\% vs 9.7\%, $P=0.002$ ) (Fig. 2).

After controlling for age, gender, BMI, smoking, baseline lung

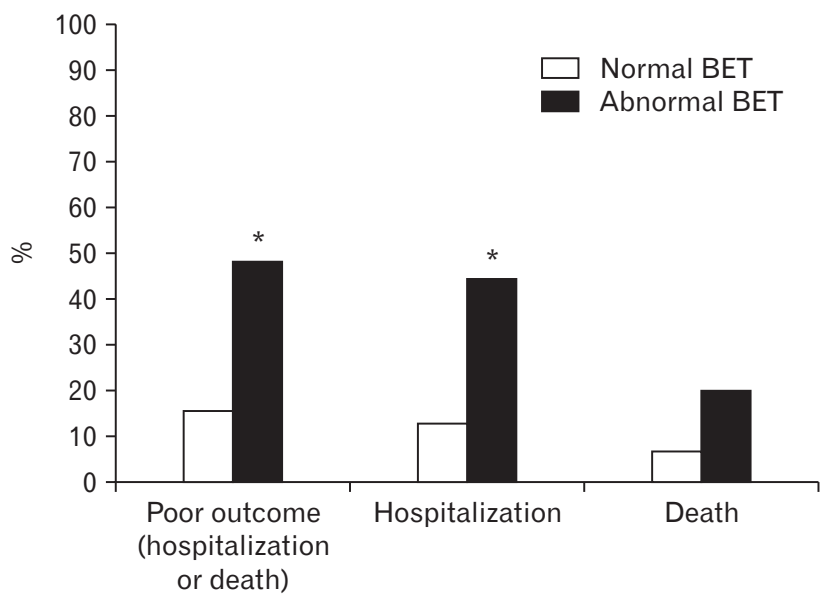

Figure 2. Incidence of poor idiopathic pulmonary fibrosis pulmonary outcome between patients with normal and abnormal bolus exposure time (BET) over 1 year. ${ }^{*} P<0.05$.

Table 1. Baseline Characteristics Between Subjects With Normal Reflux and Abnormal Reflux as Determined by Bolus Exposure Time

\begin{tabular}{|c|c|c|c|}
\hline & Normal reflux $(\mathrm{n}=38)$ & Abnormal reflux $(\mathrm{n}=46)$ & $P$-value \\
\hline Mean age (yr) & $63.1 \pm 8.94$ & $59.4 \pm 8.14$ & 0.051 \\
\hline Male gender & $26(68.4 \%)$ & $28(60.9 \%)$ & 0.472 \\
\hline On PPI prior to MII-pH study & $14(36.8 \%)$ & $33(71.7 \%)$ & 0.001 \\
\hline \multicolumn{4}{|l|}{ BMI } \\
\hline Normal $(<25)$ & $5(13.2 \%)$ & $7(15.2 \%)$ & 0.788 \\
\hline Overweight $(\geq 25)$ & $33(86.8 \%)$ & $39(84.8 \%)$ & \\
\hline Smoking history & $23(60.5 \%)$ & $31(67.4 \%)$ & 0.513 \\
\hline \multicolumn{4}{|l|}{ Baseline lung disease severity } \\
\hline \multicolumn{4}{|l|}{ DLCO } \\
\hline Mean DLCO (mL/min/mmHg) & $8.01 \pm 3.56$ & $8.12 \pm 3.30$ & 0.916 \\
\hline Moderate disease (40-60\% predicted) & $5(13.2 \%)$ & $9(19.6 \%)$ & 0.433 \\
\hline Severe disease $(<40 \%$ predicted $)$ & $33(86.8 \%)$ & $37(80.4 \%)$ & \\
\hline \multicolumn{4}{|l|}{ Oxygen requirement } \\
\hline No oxygen & $7(25.0 \%)$ & $7(17.9 \%)$ & 0.445 \\
\hline Intermittent oxygen & $13(46.4 \%)$ & $15(38.5 \%)$ & \\
\hline Continuous oxygen & $8(28.6 \%)$ & $17(43.6 \%)$ & \\
\hline
\end{tabular}

PPI, proton-pump inhibitor; BMI, body mass index; DLCO, diffusion lung capacity of oxygen; FVC, forced vital capacity. 
Table 2. Multivariate Analysis With Logistic Regression Adjusting for Age, Body Mass Index, Smoking History, and Baseline Lung Disease Severity Demonstrated That Increased Bolus Exposure Time (per Multichannel Intraluminal Impedance) Was Independently Associated With 1-Year Poor Pulmonary Outcome

\begin{tabular}{lcc}
\hline \multicolumn{1}{c}{ Co-variates } & Odds ratio & $P$-value \\
\hline Abnormal BET & 4.18 & 0.039 \\
Age & 0.97 & 0.403 \\
Male & 1.92 & 0.334 \\
Overweight (BMI $\geq 25)$ & 0.46 & 0.345 \\
Smoking History & 0.63 & 0.468 \\
PPI use & 1.65 & 0.468 \\
Severe IPF (DLCO < 40\% predicted) & 1.40 & 0.693 \\
\hline
\end{tabular}

BET, bolus exposure time; BMI, body mass index; PPI, proton pump inhibitor; IPF, idiopathic pulmonary fibrosis; DLCO, diffusion lung capacity of oxygen.

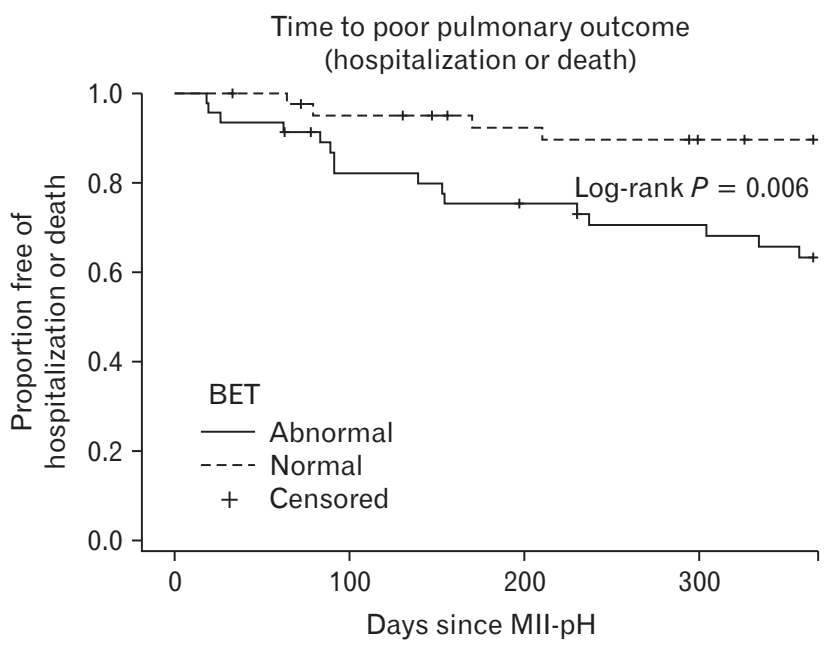

Figure 3. Kaplan-Meier analysis showing the association between abnormal bolus exposure time (BET) and decreased time to poor pulmonary outcome. MII-pH, multichannel intraluminal impedance and $\mathrm{pH}$ measurement.

disease severity, and PPI use on multivariate analysis, increased BET remained an independent predictor for poor pulmonary outcome within 1 year $(\mathrm{OR}, 4.18 ; P=0.030)$ (Table 2). Acid exposure time, as measured by $\mathrm{pH}$-monitoring, did not show similar association with poor pulmonary outcomes (OR, $0.55 ; P=0.420$ ). Subgroup analyses showed that increased BET remained an independent predictor for 1-year pulmonary hospitalization (OR, 8.26; $P=0.010$ ) when looking only at this endpoint.
Table 3. Multivariate Analysis With Cox Regression Adjusting for Age, Body Mass Index, Smoking History, and Baseline Lung Disease Severity Demonstrated That Increased Bolus Exposure Time (per Multichannel Intraluminal Impedance) Was Independently Associated With Decreased Time to Poor Pulmonary Outcome

\begin{tabular}{lcc}
\hline \multicolumn{1}{c}{ Co-variates } & Hazard ratio & $P$-value \\
\hline Abnormal BET & 4.88 & 0.007 \\
Age & 0.98 & 0.487 \\
Male & 1.26 & 0.619 \\
Overweight $($ BMI $\geq 25)$ & 0.50 & 0.162 \\
Smoking History & 0.52 & 0.159 \\
PPI use & 1.19 & 0.742 \\
Severe IPF (DLCO $<40 \%$ predicted) & 1.26 & 0.695 \\
\hline
\end{tabular}

BET, bolus exposure time; BMI, body mass index; PPI, proton pump inhibitor; IPF, idiopathic pulmonary fibrosis; DLCO, diffusion lung capacity of oxygen.

\section{Multichannel Intraluminal Impedance and $\mathrm{pH}$ Measurement and Time to Poor Pulmonary Outcome}

On univariate time-to-event analysis, increased BET was associated with a significantly decreased time to poor pulmonary outcome. This association was demonstrated on Kaplan-Meier analysis (log-ranked $P=0.002$ ) (Fig. 3). There was also a significant association between abnormal BET and decreased time to hospitalization only.

After controlling for potential confounders on multivariate Cox regression analysis, abnormal BET remained predictive of a decreased time to poor pulmonary outcome (hazard ratio [HR], 4.88; $P=0.007$ ) (Table 3 ). Acid exposure time was again not a significant predictor of decreased time to poor pulmonary outcome (HR, 0.91; $P=0.840)$. Subgroup analyses showed that increased BET remained an independent predictor of decreased time to hospitalization ( $\mathrm{HR}, 4.00 ; P=0.020$ ), when looking at this endpoint alone. A positive trend was again found between increased BET and decreased time to death $(\mathrm{HR}, 2.19 ; P=0.380)$.

\section{Discussion}

Although previous cross-sectional studies have established an association between IPF and GER, the impact of GER on the clinical course of disease in IPF patients remains unclear. Our study sought to address this question by determining whether abnormal GER as measured by MII-pH conferred a higher likelihood of poor clinical outcome over a 1-year follow-up period. We found that abnormal GER as measured by an increased BET was indeed 
a risk factor for poor pulmonary outcome, defined as either the need for hospitalization for pulmonary exacerbation or death, in this population. This association remained true even after controlling for multiple baseline clinical factors, including lung disease severity. While future prospective trials are needed to confirm a causal relationship, our findings would support the role of routine reflux assessment in patients with IPF, and consideration of aggressive antireflux therapies.

\section{Gastroesophageal Reflux Prevalence in Idiopathic Pulmonary Fibrosis}

Both acid and non-acid GER have previously been observed to be more prevalent among patients with IPF than in the healthy or non-IPF population. ${ }^{2,3}$ The findings in our IPF cohort are consistent with this observation, as we found a high prevalence of increased acid and non-acid reflux. Prior observational studies have also suggested a positive correlation between IPF disease severity and more significant GER. In 2013, Savarino et $\mathrm{al}^{1}$ found that the severity of lung fibrosis on CT scans increased with the number of reflux episodes measured in IPF patients. The same group also observed higher than normal concentrations of pepsin and bile acids in the bronchoalveolar lavage and saliva samples of their IPF patients. This led to speculation that both acid and non-acid reflux might play a role in disease pathogenesis. Another study published by our group also demonstrated that increased bolus reflux on MII-pH correlated with more abnormal pulmonary function test results. ${ }^{7}$ However, like others that previously attempted to correlate IPF severity with GER, these cross-sectional studies were unable to establish whether the presence of GER impacts or predicts the disease course of IPF. Moreover, observational studies published to date evaluating the benefits of acid suppression or anti-reflux therapies in IPF outcomes have yielded mixed results. ${ }^{8-11}$ The role of GER evaluation and treatment in the management of IPF, therefore, could not be conclusively established based on currently available evidence. Despite the lack of supportive outcome data, the American Thoracic Society has recommended the use of PPI in the care of IPF patients in its newest consensus management guidelines. ${ }^{12}$ Our study and results may help provide evidence to address this question by demonstrating that abnormal bolus reflux at baseline is independently predictive of worse outcomes from pulmonary disease over 1 year.

\section{Predictive Factors in Idiopathic Pulmonary Fibrosis}

Several risk factors for poor outcome in IPF have previously been described, including age, ${ }^{13}$ respiratory hospitalization, ${ }^{13,14}$ a decline in forced vital capacity (FVC), ${ }^{13,15,16}$ and a decline in diffusion capacity of the lungs for carbon monoxide (DLCO) ${ }^{13}$ In 2012, Ley et $\mathrm{al}^{17}$ found that a composite score calculated from a patient's age, gender, FVC, and DLCO was predictive of 1-year mortality. This model was subsequently improved by the addition of longitudinal variables including history of respiratory hospitalizations and a 24-week change in FVC. ${ }^{18}$ To our knowledge, ours is the first study to examine the association between objective GER parameters and poor clinical outcomes in this population. We found that an abnormally increased BET was a significant predictor of poor composite outcome after 1 year, and importantly, this remained true after controlling for several of these previously identified risk factors, including age, gender, and baseline lung disease severity (as determined by DLCO in our model). In addition, our results remained significant after controlling for BMI, smoking history, and PPI use.

We believe that our findings support the adoption of reflux testing in the clinical care of IPF patients. Given the current paucity of effective treatments for IPF and the unpredictable nature of the illness, it is important to address all potentially modifiable risk factors that may improve prognosis. The results from our cohort strengthen the theory that reflux may contribute to IPF pathogenesis, and that identifying and aggressively treating significant reflux in this population may help to slow disease progression.

Routine reflux testing with MII-pH in this population may also serve as a useful prognostic tool. Our results show that IPF patients with abnormal bolus reflux suffer higher rates of hospitalization and death, and at an earlier time, than those with physiologic reflux. Abnormal findings on MII-pH may, therefore, serve as a marker of accelerated disease progression and increased complications. Identifying such patients may allow for more intensive clinical management, including, but not limited to, earlier consideration of lung transplantation.

\section{Bolus Versus Acid Reflux}

The relative importance of acid versus bolus reflux in IPF patients is not known, but recent evidence suggests that impedance measures of bolus reflux may be a better predictor of fibrosis and disease severity compared to $\mathrm{pH}$ measures of acid reflux alone. ${ }^{1,3,7}$ Our current findings that abnormal bolus reflux is a significant risk factor for poor pulmonary outcome in IPF is consistent with this idea. We also did not find a significant association between acid reflux parameters alone and poor outcome. The lack of correlation between acid parameters alone such as AET and poor pulmonary outcome may be due to two main reasons. First, patients with ab- 
normal AET may have more likely been maintained on PPI after MII-pH in this cohort. PPI may exert a protective effect in IPF patients through reflux burden reduction, but it has also been speculated to carry an anti-inflammatory effect through suppression of pro-inflammatory cells and molecules. ${ }^{19}$ Prior studies using animal models of lung injury showed that PPI may decrease lung inflammatory and fibrotic responses by mediating pro-inflammatory and pro-fibrotic factors. ${ }^{20}$ Second, the $\mathrm{pH}$ definition of what constitutes acid reflux, namely a $\mathrm{pH}$ cutoff of 4 , was established and validated for esophageal symptoms and injury. Whether this definition similarly applies to airway or pulmonary complications is less clear. Weakly acidic ( $\mathrm{pH}$ between 4 and 7$)$ and weakly alkaline $(\mathrm{pH}>7)$ reflux events may also contribute to airway and pulmonary injury, thus leading to worse clinical outcome. Acid based parameters using the tradition $\mathrm{pH}<4$ cutoff may, therefore, underestimate the true reflux burden and risk in the context of pulmonary disease.

There is prior observational data to suggest that use of acid suppression in IPF patients may improve outcomes. In 2011, Lee et $\mathrm{al}^{21}$ reported that the use of acid suppression among a group of IPF patients was associated with longer survival time and lower fibrosis scores on CT scan, though this conclusion was based solely on patient reporting of medication use. In a subsequent study, the same group observed that the use of acid suppression in IPF patients led to a slower decline in FVC and fewer acute exacerbations. ${ }^{8}$ The data for that study was drawn from the pooled placebo arms of three separate trials designed to evaluate other IPF therapies. Results from other studies did not consistently show similar beneficial effects of acid suppression in this population. ${ }^{10,11}$ However, many of these results were extracted from secondary outcomes of larger IPF studies. Our study suggests that acid suppression may provide some benefit to IPF patients, perhaps most measurably to those with abnormal acid reflux. Given the high prevalence of non-acid reflux as shown in this and prior studies, and the significant predictive value of BET, but not AET, for poor outcome, anti-reflux therapy such as fundoplication may be more beneficial compared to acid suppression alone to improve clinical outcomes in this population. Such therapeutic implication would need further prospective trials to better ascertainment, but our data would support the potential role of MII-pH in directing both the need for and the type of reflux therapy used in this population.

\section{Limitations}

The primary limitation of our study is its retrospective design, although patients at the pulmonary/IPF center of our institution are generally managed following a standard protocol. Moreover, our lung transplant center requires all patients to undergo MII-pH as part of the pre-transplant evaluation regardless of presence of reflux symptoms, thereby minimizing potential selection bias. Although our study is also limited by sample size, to our knowledge this is one of the largest IPF cohorts to date analyzing objective MII-pH reflux parameters. Since we collected data on patients over several years spanning from June 2008 to January 2015, our results are subject to secular changes in IPF treatment during this time. While the use of common therapies such as corticosteroids may have varied on an individual basis during the study period, the true value of these therapies in slowing IPF disease progression remains uncertain per previous published data. Therefore, we do not believe that this would significantly impact our results. The use of pirfenidone is one novel therapeutic that has recently been shown to potentially reduce disease progression and improve survival. ${ }^{22}$ However, since this novel treatment was not yet widely adopted at our center during the study period, this was not relevant to our cohort. Finally, the phenomenon of reverse causation is an important consideration for any study investigating reflux in pulmonary patients, as it is not known with certainty whether abnormal reflux or alteration in thoracic mechanics due to underlying respiratory disease serves as the main driver. We sought to minimize this type of bias by controlling for baseline lung disease severity in our model.

In conclusion, we found that abnormally increased BET on pre-lung transplant MII-pH is an independent predictor of poor pulmonary outcome over 1 year in IPF patients after controlling for multiple baseline variables including pulmonary function. This supports the idea that GER may play a role in IPF pathogenesis in some patients, and that routine reflux assessment with MII-pH may have clinical value in the management of these patients. Future prospective trials are needed to confirm a causal relationship and to further explore the therapeutic benefits of anti-reflux therapy in IPF patients. In addition, investigators designing clinical trials in IPF should control for abnormal reflux to avoid confounding. Given the current paucity of treatment options for IPF, we believe that routine MII-pH should be considered to identify candidates for aggressive anti-reflux therapy in further clinical management of IPF.

\section{Financial support: None.}

\section{Conflicts of interest: None.}

Author contributions: Walter W Chan and Lawrence F Borges initiated study concepts and design; Lawrence F Borges, Vikrant Jagadeesan, Hilary Goldberg, Sravanya Gavini, and Natan 
Feldman contributed to acquisition of data; Walter W Chan, Lawrence F Borges, Hilary Goldberg, Sravanya Gavini, Wai-Kit Lo, and Robert Burakoff performed analysis and interpretation of data; Walter W Chan and Lawrence F Borges drafted the manuscript; Walter W Chan, Lawrence F Borges, Sravanya Gavini, Wai-Kit Lo, Hilary Goldberg, and Robert Burakoff contributed to critical revision of manuscript for important intellectual content; Walter W Chan and Lawrence F Borges performed statistical analyses; and Walter W Chan provided administrative support and overall study supervision.

\section{References}

1. Savarino E, Carbone R, Marabotto E, et al. Gastro-oesophageal reflux and gastric aspiration in idiopathic pulmonary fibrosis patients. Eur Respir J 2013;42:1322-1331.

2. Tobin RW, Pope CE 2nd, Pellegrini CA, Emond MJ, Sillery J, Raghu G. Increased prevalence of gastroesophageal reflux in patients with idiopathic pulmonary fibrosis. Am J Respir Crit Care Med 1998;158:1804-1808.

3. Gavini S, Finn RT, Lo WK, et al. Idiopathic pulmonary fibrosis is associated with increased impedance measures of reflux compared to nonfibrotic disease among pre-lung transplant patients. Neurogastroenterol Motil 2015;27:1326-1332.

4. Raghu G, Collard HR, Egan JJ, et al. An official ATS/ERS/JRS/ALAT statement: idiopathic pulmonary fibrosis: evidence-based guidelines for diagnosis and management. Am J Respir Crit Care Med 2011;183:788824.

5. Hirano I, Richter JE; Practice Parameters Committee of the American College of Gastroenterology. ACG practice guidelines: esophageal reflux testing. Am J Gastroenterol 2007;102:668-685.

6. Shay S, Tutuian R, Sifrim, et al. Twenty-four hour ambulatory simultaneous impedance and $\mathrm{pH}$ monitoring: a multicenter report of normal values from 60 healthy volunteers. Am J Gastroenterol 2004:99:1037-1043.

7. Gavini S, Borges LF, Finn RT, et al. Lung disease severity in idiopathic pulmonary fibrosis is more strongly associated with impedance measures of bolus reflux than $\mathrm{pH}$ parameters of acid reflux alone. Neurogastroenterol Motil 2017;29:e13001.

8. Lee JS, Collard HR, Anstrom KJ, et al. Anti-acid treatment and disease progression in idiopathic pulmonary fibrosis: an analysis of data from three randomised controlled trials. Lancet Respir Med 2013;1:369-376.

9. Lee JS, Ryu JH, Elicker BM, et al. Gastroesophageal reflux therapy is associated with longer survival in patients with idiopathic pulmonary fibrosis. Am J Respir Crit Care Med 2011;184:1390-1394.

10. Kreuter M, Wuyts W, Renzoni E, et al. Antacid therapy and disease outcomes in idiopathic pulmonary fibrosis: a pooled analysis. Lancet Respir Med 2016;4:381-389.

11. Kreuter M, Spagnolo P, Wuyts W, et al. Antacid therapy and disease progression in patients with idiopathic pulmonary fibrosis who received pirfenidone. Respiration 2017;93:415-423.

12. Raghu G, Rochwerg B, Zhang Y, et al. An Official ATS/ERS/JRS/ ALAT clinical practice guideline: treatment of idiopathic pulmonary fibrosis. An update of the 2011 clinical practice guideline. Am J Respir Crit Care Med 2015;192:e3-e19.

13. Du Bois RM, Weycker D, Albera C, et al. Ascertainment of individual risk of mortality for patients with idiopathic pulmonary fibrosis. Am J Respir Crit Care Med 2011;184:459-466.

14. Brown AW, Fischer CP, Shlobin O a, et al. Outcomes after hospitalization in idiopathic pulmonary fibrosis: a cohort study. Chest 2015;147:173179.

15. King TE, Safrin S, Starko KM, et al. Analyses of efficacy end points in a controlled trial of interferon-gammalb for idiopathic pulmonary fibrosis. Chest 2005;127:171-177.

16. Collard HR, King TE Jr, Bartelson BB, Vourlekis JS, Schwarz MI, Brown KK. Changes in clinical and physiologic variables predict survival in idiopathic pulmonary fibrosis. Am J Respir Crit Care Med 2003;168: 538-542.

17. Ley B, Ryerson CJ, Vittinghoff E, et al. A multidimensional index and staging system for idiopathic pulmonary fibrosis. Ann Intern Med 2012; 156:684-691.

18. Ley B, Bradford WZ, Weycker D, Vittinghoff E, du Bois RM, Collard HR. Unified baseline and longitudinal mortality prediction in idiopathic pulmonary fibrosis. Eur Respir J 2015;45:1374-1381.

19. Kedika RR, Souza RF, Spechler SJ. Potential anti-inflammatory effects of proton pump inhibitors: a review and discussion of the clinical implications. Dig Dis Sci 2009;54:2312-2317.

20. Ghebremariam YT, Cooke JP, Gerhart W, et al. Pleiotropic effect of the proton pump inhibitor esomeprazole leading to suppression of lung inflammation and fibrosis. J Transl Med 2015;13:249.

21. Lee JS, Ryu JH, Elicker BM, et al. Gastroesophageal reflux therapy is associated with longer survival in patients with idiopathic pulmonary fibrosis. Am J Respir Crit Care Med 2011;184:1390-1394.

22. King TE, Bradford WZ, Castro-Bernardini S, et al. A Phase 3 Trial of Pirfenidone in Patients with Idiopathic Pulmonary Fibrosis. N Engl J Med 2014;370:2083-2092. 